\title{
Cargas polínicas de abelhas polinizadoras de Byrsonima chrysophylla Kunth. (Malpighiaceae): fidelidade e fontes alternativas de recursos florais ${ }^{1}$
}

\author{
Éville Karina Maciel Delgado Ribeiro ${ }^{2,4}$, Márcia Maria Corrêa Rêgo² e Isabel Cristina Sobreira Machado³
}

Recebido em 26/02/2007. Aceito em 23/05/2007

\begin{abstract}
RESUMO - (Cargas polínicas de abelhas polinizadoras de Byrsonima chrysophylla Kunth. (Malpighiaceae): fidelidade e fontes alternativas de recursos florais). Byrsonima chrysophylla, conhecida popularmente como murici pitanga, é uma espécie arbustiva que ocorre em restinga. Dentre seus visitantes, além de abelhas da tribo Centridini, também se encontram com freqüência abelhas Xylocopini. Para verificação das espécies vegetais utilizadas pelas abelhas visitantes mais freqüentes, cargas polínicas de suas pernas posteriores $(\mathrm{n}=5)$, bem como de ninhos naturais de Centris caxiensis $(\mathrm{n}=7)$ foram analisados através de acetólise. Durante toda a floração, Byrsonima chrysophylla foi a principal fonte de pólen utilizada por suas abelhas visitantes. Comolia lythrarioides e Myrcia obtusa foram fontes secundárias de pólen, Mouriri guianensis foi fonte secundária de óleo e pólen, enquanto Anacardium microcarpum, Cuphea tenella, Matayba discolor e Phthirusa pyrifolia mostraram-se importantes fontes de néctar durante esse período, sendo o pólen detectado nas amostras, provavelmente aderido ao corpo das abelhas durante a coleta de néctar. Os resultados demonstram que as abelhas visitantes não misturaram os recursos polínicos durante o vôo para a coleta de pólen, apresentando fidelidade às flores de Byrsonima chrysophylla.
\end{abstract}

Palavras-chave: Byrsonima, pólen, Centris, Xylocopa, fidelidade polínica, restinga

\begin{abstract}
Pollen loads of pollinator bees of Byrsonima chrysophylla Kunth. (Malpighiaceae): fidelity and alternative sources of flower resources). Byrsonima chrysophylla, known as "murici pitanga", is a shrubby species occurring in 'restinga'. Among its visitors, bees of the Centridini tribe as well as Xylocopini bees are frequently found. To detect which plant species have been used by the most frequent visitors, pollen loads from the back legs $(n=5)$ and also from natural nests of Centris caxiensis $(n=7)$ were treated by acetolysis. During flowering of Byrsonima chrysophylla, this plant species was the main pollen source used by bees. Comolia lythrarioides and Myrcia sylvatica were secondary pollen sources, Mouriri guianensis was a secondary oil and pollen source, while Anacardium microcarpum, Cuphea tenella, Matayba discolor and Phthirusa pyrifolia were important nectar sources at this time. The pollen detected in the samples probably adhered to the bee's body during nectar collection. The results show that the bee visitors do not mix pollen sources during the pollen-collecting flight, thus representing fidelity to Byrsonima chrysophylla flowers.
\end{abstract}

Key works: Byrsonima, pollen, Centris, Xylocopa, pollen fidelity, 'restinga'

\section{Introdução}

Malpighiaceae é uma família de eudicotiledôneas tropicais e subtropicais, com mais de 1.500 espécies distribuídas em cerca de 65 gêneros (Cameron et al. 2001). Destas, $85 \%$ são endêmicas da região Neotropical (Anderson 1979) com o centro de diversidade no norte da América do Sul (Anderson 1981). No Brasil ocorrem 38 gêneros, com cerca de 300 espécies, distribuídas nas mais diversas regiões (Souza \& Lorenzi 2005).
Enquanto no Velho Mundo apenas 52\% das espécies de Malpighiaceae oferecem óleo aos polinizadores, a maioria das espécies neotropicais desta família possui glândulas de óleo, denominadas elaióforos (Vogel 1990). Contudo, é importante ressaltar que, embora o óleo seja o principal recurso oferecido pela maioria das espécies neotropicais de Malpighiaceae, o pólen também atrai uma grande diversidade de visitantes que, mesmo secundariamente, podem atuar como polinizadores (Pedro 1994).

\footnotetext{
Parte da Dissertação de Mestrado da primeira Autora

2 Universidade Federal de Maranhão, Departamento de Biologia, Centro de Ciências Biológicas e da Saúde, Avenida dos Portugueses, s.n., Campus Universitário do Bacanga, 65085-580 São Luís, MA, Brasil

3 Universidade Federal de Pernambuco, Departamento de Botânica, Centro de Ciências Biológicas, Rua Prof. Moraes Rego, s.n., Cidade Universitária, 50372-970 Recife, PE, Brasil

4 Autora para correspondência: evillekarina@yahoo.com.br
} 
Os lipídeos florais são utilizados pelas abelhas na construção de ninhos (Vinson et al. 1997) e, misturados com o pólen, formam um bolo alimentar usualmente chamado de "pão de mel", que é utilizado como alimento larval (Vogel 1974; Simpson \& Neff 1981; Rêgo \& Albuquerque 2004; Rêgo et al. 2006) e, possivelmente, na nutrição de adultos (Buchmann 1987).

O pólen tem duas funções principais nos ecossistemas: é essencial para a reprodução das plantas e é utilizado como recompensa para visitantes florais ou polinizadores. Abelhas fêmeas coletam grandes quantidades de pólen das flores e servem como alimento para suas larvas. Embora este pólen não seja usado na polinização, ele é utilizado na alimentação de futuros polinizadores (Schlindwein et al. 2005).

A composição das cargas polínicas de abelhas fêmeas é como a "impressão digital" de seus hábitos de forrageamento e, analisando a qualidade do conteúdo polínico encontrado nas corbículas e/ou escopas dessas abelhas podemos inferir suas eficiências como polinizadoras de certas espécies de plantas (Wittmann \& Schlindwein 1995). A identificação de vários tipos de pólen nas pernas e nos ninhos das abelhas é de fundamental importância, pois indica as plantas que estas utilizaram para a coleta de pólen e néctar (ou óleo, no caso das Centridini) e nos permite avaliar a importância de cada uma das plantas visitadas, como fornecedoras de recursos para seus polinizadores (Eckert 1942).

Byrsonima chrysophylla Kunth. é popularmente conhecida na região de Barreirinhas, Maranhão, como "murici pitanga". É uma planta de porte arbustivo, com altura variando de 1 a 1,25 metros e possui curto período de floração, que ocorre do final de janeiro a início de março, caracterizado pela produção massiva de flores (Ribeiro et al. 2006).

As flores de representantes do gênero Byrsonima são visitadas por vários grupos de Apoidea (Albuquerque \& Rêgo 1989; Barros 1992; Teixeira \& Machado 2000; Rêgo \& Albuquerque 2004; 2006; Machado 2004; Costa et al. 2006), porém, apenas abelhas das tribos Centridini, Tapinotaspidini e Tetrapediini coletam óleo ativamente, rompendo os elaióforos por meio de fricção de estruturas especializadas presentes em suas pernas anteriores e medianas.

Este trabalho teve como objetivo determinar a freqüência dos visitantes florais de Byrsonima chrysophylla e analisar suas cargas polínicas, a fim de verificar a importância desta espécie vegetal em relação a outras espécies vegetais para a sobrevivência de seus polinizadores.

\section{Material e métodos}

Este estudo foi realizado do final de janeiro a início de março/2006, numa reserva particular de restinga, na Fazenda Buriti, situada no povoado de Cantinho, no Município de Barreirinhas, Maranhão ( $2^{\circ} 43^{\prime 22,5 " S}$; 42 49'50"W) que dista $345 \mathrm{~km}$ da capital do Estado, São Luís (IBGE 1984). O município de Barreirinhas está incluído na área de influência do Parque Nacional dos Lençóis Maranhenses.

A vegetação do Parque Nacional dos Lençóis Maranhenses ocupa uma área de 453,28 $\mathrm{km}^{2}$ dos quais $405,16 \mathrm{~km}^{2}$ são compostos predominantemente por restinga, correspondendo a $89 \%$ da área total. Ocorrem ainda os manguezais e as comunidades aluviais (matas ciliares), que correspondem, respectivamente a apenas 10,2\% e 4\% da vegetação total (MMA/IBAMA 2002).

O clima da região onde se situa a área é tropical megatérmico (enquadrando-se no tipo Aw', de acordo com a classificação de Köppen), com pluviosidade anual em torno de $1.800 \mathrm{~mm}$, a maior parte das chuvas estando concentrada nos meses de dezembro a maio, com os meses de julho a outubro muito secos. A temperatura média anual varia entre $26{ }^{\circ} \mathrm{C}$ e $27^{\circ} \mathrm{C}$ (BRASIL 1984).

Estudos realizados por Rêgo e colaboradores (dados não publicados) verificaram a ocorrência de 83 morfoespécies vegetais na área, das quais 67 foram identificadas, estando distribuídas em 31 famílias, sendo as mais representativas, em número de espécies, Caesalpinaceae $(n=8)$, Fabaceae $(n=6)$, Myrtaceae $(\mathrm{n}=4)$, Rubiaceae $(\mathrm{n}=4)$ e Malpighiaceae $(\mathrm{n}=4)$.

As coletas dos visitantes florais foram feitas de forma periódica, em intervalos de sete dias, das $6 \mathrm{~h}$ às $17 \mathrm{~h}$, totalizando $55 \mathrm{~h}$ de amostragem. Os visitantes florais foram coletados após visita às flores de B. chrysophylla, com auxílio de rede entomológica. Em seguida, foram montados, identificados e depositados na coleção do Laboratório de Estudos sobre Abelhas da Universidade Federal do Maranhão.

As coletas foram divididas de acordo com a intensidade e quantidade de flores de B. chrysophylla em: início (final do mês de janeiro a início de fevereiro), pico (meados de fevereiro) e final da floração (final de fevereiro a início de março).

Para verificação das outras espécies vegetais utilizadas pelas abelhas visitantes mais freqüentes de B. chrysophylla, foram realizadas análises das cargas polínicas de suas pernas posteriores $(\mathrm{n}=5$, para cada espécie de abelha), bem como de ninhos naturais $(\mathrm{n}=7)$ de Centris caxiensis (Ducke 1907), única espécie que 
foi encontrada nidificando no sítio de estudo durante este período.

As cargas polínicas foram retiradas das pernas posteriores das abelhas com auxílio de estiletes. Os ninhos de Centris caxiensis foram marcados e, quando finalizados, escavados, retirando-se suas células. As células foram abertas e seu conteúdo polínico extraído.

O material obtido das pernas posteriores das abelhas e dos ninhos foi submetido ao processo de acetólise (Erdtman 1966). De cada amostra foram confeccionadas cinco lâminas, sendo contados 100 grãos de pólen de cada uma delas, totalizando 500 grãos por amostra. Em seguida, determinou-se as porcentagens e classes de ocorrência de acordo com classificação de Louveaux et al. (1978) em: pólen dominante ( $>45 \%$ do total de grãos), pólen acessório (de $15 \%$ a $45 \%$ ), pólen isolado importante (de $3 \%$ a $14 \%)$ e pólen isolado ocasional $(<3 \%)$.

Os grãos de pólen encontrados foram classificados em tipos polínicos, de acordo com sua morfologia. Posteriormente, foram fotografados e a identificação botânica das cargas polínicas realizou-se mediante comparação da morfologia dos grãos de pólen obtidos com a dos grãos de pólen da palinoteca referência da área, depositada no Laboratório de Estudos sobre Abelhas, da Universidade Federal do Maranhão. Algumas lâminas foram enviadas a especialista para confirmação da identificação.

\section{Resultados e discussão}

As flores de Byrsonima chrysophylla são visitadas por 16 espécies de abelhas, pertencentes às tribos Centridini, Tapinotaspidini, Meliponini e Xylocopini (Tab. 1).

Centridini é o grupo de abelhas coletoras de lipídios florais mais diversificado e restrito às Américas, onde Malpighiaceae aparece como a principal família produtora deste recurso (Buchmann 1987; Vogel 1990; Ramalho \& Silva 2002). As abelhas do gênero Centris são restritas às regiões Neotropical e Neártica, ocorrendo desde regiões áridas até florestas úmidas equatoriais (Zanella 2002), sendo 97 espécies diagnosticadas no Brasil (Silveira et al. 2002).

Diversos trabalhos descrevem as abelhas da tribo Centridini como as polinizadoras mais eficazes do gênero Byrsonima (ver Machado 2004 para revisão). Em B. chrysophylla não é diferente, sua polinização é feita quase que exclusivamente por algumas espécies de Centridini, embora também possa ser realizada por uma espécie de Xylocopini (Ribeiro et al. 2006).
Tabela 1. Espécies de abelhas visitantes florais de Byrsonima chrysophylla e freqüência de suas visitas em restinga no município de Barreirinhas, MA, Brasil, no período de janeiro a março/2006, organizadas pela ordem de freqüência dentro de cada tribo.

\begin{tabular}{lc}
\hline Tribo/Espécies & Frequiência (\%) \\
\hline Centridini & \\
Centris caxiensis (Ducke, 1907) & 30,00 \\
C. aenea (Lepeletier, 1941) & 14,14 \\
C. leprieuri (Spinola, 1941) & 8,27 \\
C. byrsonimae In litt. & 4,83 \\
C. tarsata (Smith, 1874) & 2,41 \\
C. sponsa (Smith, 1854) & 1,03 \\
C. longimana (Fabricius, 1804) & 0,69 \\
Epicharis umbraculata (Fabricius, 1804) & 0,69 \\
Centris analis (Fabricius, 1804) & 0,34 \\
C. flavifrons (Fabricius, 1775) & 0,34 \\
Tapinotaspidini & \\
Paratetrapedia globulosa (Friese, 1899) & 0,69 \\
Meliponini & \\
Trigona fulviventris (Guérin, 1835) & 24,48 \\
Trigonisca extrema In litt. & 3,10 \\
Melipona flavolineata (Friese, 1900) & 2,07 \\
Xylocopini & \\
Xylocopa cearensis (Ducke, 1910) & 6,90 \\
X. frontalis (Olivier, 1789) & 0,69 \\
\hline
\end{tabular}

A tribo Xylocopini, abundante nas regiões tropicais e subtropicais, é representada apenas pelo gênero Xylocopa, o qual apresenta cerca de 50 espécies no Brasil (Silveira et al. 2002). Visitas de abelhas Xylocopini a flores que possuem óleo como atrativo primário também foram relatadas por Freitas et al. (1999), em Malpighia emarginata, e por Benezar \& Pessoni (2006), em Byrsonima coccolobifolia. No entanto, os autores não as reconhecem como polinizadoras destas "plantas de óleo".

A análise das cargas polínicas de Centris caxiensis, Centris leprieuri (Spinola, 1941), Centris aenea (Lepeletier, 1941) e Xylocopa cearensis (Ducke, 1910), visitantes mais freqüentes e prováveis polinizadoras de $B$. chrysophylla, demonstrou a presença de oito tipos polínicos (Fig. 1-8), pertencentes a oito espécies vegetais, distribuídas em sete famílias e oito gêneros (Tab. 2). Em todas as amostras de cargas polínicas, B. chrysophylla foi predominante (Tab. 3, 4).

Durante o início da floração de B. chrysophylla, grãos de pólen de Cuphea tenella Hook. \& Arn. (Lythraceae) e Matayba discolor Radlk (Sapindaceae) foram isolados ocasionais nas cargas polínicas das pernas posteriores de Centris caxiensis, enquanto que no pico da floração, apenas grãos de pólen de B. chrysophylla foram encontrados. No final 
da floração, grãos de Cuphea tenella foram novamente isolados ocasionais (Tab. 2).

De forma semelhante, nos ninhos de Centris caxiensis foram registrados grãos isolados ocasionais de Cuphea tenella, Myrcia obtusa Schauer (Myrtaceae), Phthirusa pyrifolia (H.B.K.) Eichl. (Loranthaceae) e Matayba discolor, enquanto grãos de pólen de B. chrysophylla foram dominantes (Tab. 4).
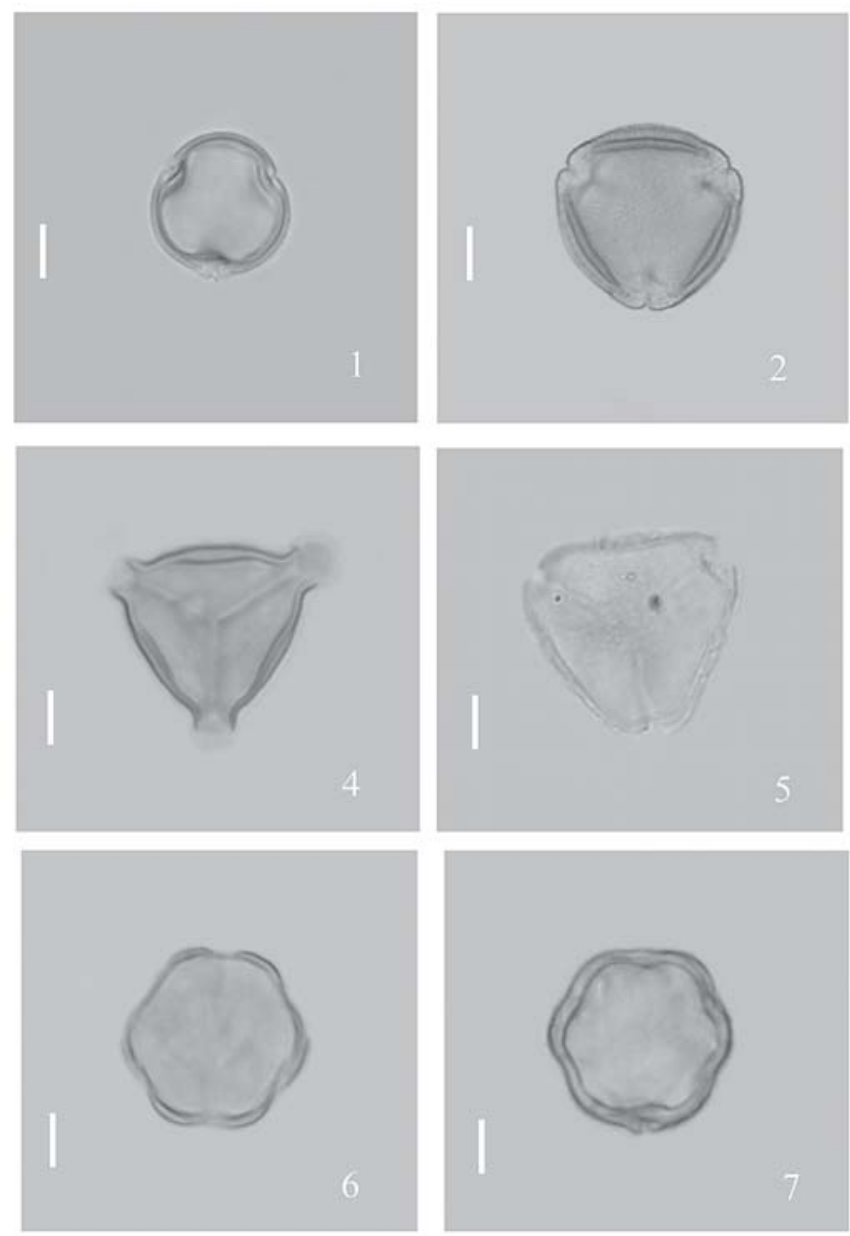

Figuras 1-8. Tipos polínicos de distintas espécies vegetais encontrados em abelhas visitantes florais de Byrsonima chrysophylla Kunth. 1. Byrsonima chrysophylla Kunth. (Malpighiaceae). 2. Anacardium microcarpum Ducke (Anacardiaceae). 3. Phthirusa pyrifolia (H.B.K.) Eichl. (Loranthaceae). 4. Cuphea tenella Hook. \& Arn. (Lythraceae). 5. Myrcia obtusa Schauer (Myrtaceae). 6. Comolia lythrarioides (Steudel) Naudin (Melastomataceae). 7. Mouriri guianensis Aubl. (Melastomataceae). 8. Matayba discolor Radlk. (Sapindaceae). Barras $=10 \mu \mathrm{m}$.

Tabela 2. Espécies vegetais identificadas por meio de análise das cargas polínicas de pernas e ninhos de abelhas visitantes de Byrsonima chrysophylla Kunth., seus nomes vulgares e recursos oferecidos aos visitantes em restinga no município de Barreirinhas, MA, Brasil. $\mathrm{N}=$ néctar, $\mathrm{P}=$ pólen e $\mathrm{O}=$ óleo.

\begin{tabular}{lll}
\hline Família & Espécie & Nome vulgar \\
\hline Anacardiaceae & Anacardium microcarpum Ducke & cajuí \\
Loranthaceae & Phthirusa pyrifolia (H.B.K.) Eichl. & cipó ritirana \\
Lythraceae & Cuphea tenella Hook. \& Arn. & vassoura \\
Malpighiaceae & Byrsonima chrysophylla Kunth. & murici pitanga \\
Melastomataceae & Comolia lythrarioides (Steudel) Naudin & malva roxa \\
& Mouriri guianensis Aubl. & puçá \\
Myrtaceae & Myrcia obtusa Schauer & branquinha \\
Sapindaceae & Matayba discolor Radlk. & orelha-de-cachorro \\
\end{tabular}


Tabela 3. Espécies vegetais e porcentagem de grãos de pólen encontrados nas pernas posteriores de Centris aenea Lepeletier (1941), C. caxiensis Ducke (1907), C. leprieuri Spinola (1941) e Xylocopa cearensis Ducke (1910), coletadas em visitas às flores de Byrsonima chrysophylla Kunth. durante o início (final do mês de janeiro a início de fevereiro/2006), pico (meados de fevereiro) e fim da sua floração (final de fevereiro a início de março) em restinga no município de Barreirinhas, MA, Brasil.

\begin{tabular}{|c|c|c|c|}
\hline \multirow[t]{2}{*}{ Abelhas visitantes/espécies vegetais } & \multicolumn{3}{|c|}{ Período de floração de Byrsonima chrysophylla } \\
\hline & Início & Pico & Final \\
\hline \multicolumn{4}{|l|}{ Centris aeneal } \\
\hline Anacardium microcarpum & 0 & 0 & 3,67 \\
\hline Byrsonima chrysophylla & 98,5 & 100 & 86,33 \\
\hline Comolia lythrarioides & 0 & 0 & 5 \\
\hline Cuphea tenella & 0,25 & 0 & 0 \\
\hline Matayba discolor & 1,25 & 0 & 0 \\
\hline Mouriri guianensis & 0 & 0 & 5 \\
\hline \multicolumn{4}{|l|}{ Centris caxiensis/ } \\
\hline Byrsonima chrysophylla & 98,25 & 100 & 99,97 \\
\hline Cuphea tenella & 0,75 & 0 & 0,03 \\
\hline Matayba discolor & 1 & 0 & 0 \\
\hline \multicolumn{4}{|l|}{ Centris leprieuri/ } \\
\hline Byrsonima chrysophylla & 100 & 100 & 100 \\
\hline \multicolumn{4}{|l|}{ Xylocopa cearensis/ } \\
\hline Byrsonima chrysophylla & 100 & 90 & 99,67 \\
\hline Comolia lythrarioides & 0 & 10 & 0,08 \\
\hline Phthirusa pyrifolia & 0 & 0 & 0,25 \\
\hline
\end{tabular}

Estudando os recursos florais disponíveis aos polinizadores de Byrsonima crassifolia, espécie simpátrica de Byrsonima chrysophylla, Silva et al. (2006) também observaram a presença de grãos de pólen de B. chrysophylla, Cuphea tenella, Myrcia obtusa e Phthirusa pyrifolia em cargas polínicas de Centris caxiensis. No estudo, realizado no período de julho a outubro na restinga do município de Barreirinhas, outras oito espécies vegetais foram encontradas nas cargas desta abelha (Silva et al. 2006).

A Tab. 3 mostra ainda as espécies vegetais utilizadas por Centris aenea. A análise da carga polínica demonstrou que, no início da floração, grãos de Matayba discolor e Cuphea tenella foram isolados ocasionais. No pico da floração, entretanto, apenas $B$. chrysophylla foi utilizada como recurso para esta abelha. No final da floração, grãos de pólen de Anacardium microcarpum Ducke (Anacardiaceae), Comolia lythrarioides (Steudel) Naudin. (Melastomataceae) e Mouriri guianensis Aubl. (Melastomataceae) foram isolados importantes, entretanto novamente os grãos de $B$. chrysophylla foram dominantes.

Por sua vez, a análise polínica das cargas das pernas posteriores de Centris leprieuri demonstrou $100 \%$ de grãos de pólen de $B$. chrysophylla durante todo o período de floração (Tab. 3).
Tabela 4. Espécies vegetais e respectiva porcentagem de grãos de pólen encontrados em ninhos naturais de Centris caxiensis Ducke, durante o mês de fevereiro/2006, em uma área de restinga em Barreirinhas, MA, Brasil.

\begin{tabular}{lc}
\hline Espécies vegetais & Grãos de pólen \\
\hline Byrsonima chrysophylla & 98,41 \\
Cuphea tenella & 0,79 \\
Matayba discolor & 0,02 \\
Myrcia obtusa & 0,71 \\
Phthirusa pyrifolia & 0,07 \\
\hline
\end{tabular}

A relação de especificidade entre abelhas do gênero Centridini e flores de Byrsonima já se encontra bem descrita quanto à coleta de óleos florais. Neste trabalho pôde-se observar também a importância quanto à utilização do pólen de flores de uma espécie de Byrsonima para abelhas desta tribo.

No início da floração, apenas $B$. chrysophylla foi utilizada como recurso para Xylocopa cearensis (Tab. 3). No pico da floração, grãos de pólen de Comolia lythrarioides foram isolados importantes, com freqüência de $10 \%$, enquanto grãos de B. chrysophylla foram dominantes. No final da floração, grãos de Phthirusa pyrifolia e Comolia lythrarioides foram isolados ocasionais e grãos de B. chrysophylla foram dominantes. 
Abelhas do gênero Xylocopa utilizam néctar e pólen como únicas fontes de alimento. Muitas das espécies encontram-se associadas a flores com anteras poricidas (Buchmann 1983), contudo, a maioria é generalista, visitando grande número de espécies vegetais, concentrando-se, porém, em alguns recursos mais produtivos (Gerling et al. 1989).

Grãos de pólen de $B$. chrysophylla constituíram mais de $90 \%$ dos registrados nas amostras de pólen obtidas de seus visitantes mais freqüentes durante toda a floração, excetuando-se as amostras de $X$. cearenses coletadas no final da floração de B. chrysophylla. Esta espécie vegetal representou a única fonte de recurso polínico para $C$. leprieuri, com $100 \%$ de ocorrência em todas as amostras, evidenciando um alto grau de fidelidade a esta fonte floral. As espécies vegetais Comolia lythrarioides e Myrcia obtusa representaram fontes secundárias de pólen, Mouriri guianensis foi, possivelmente, fonte secundária de óleo e pólen, enquanto Anacardium microcarpum, Cuphea tenella, Matayba discolor e Phthirusa pyrifolia foram importantes fontes de néctar durante o período de estudo, sendo que os grãos de pólen detectados nas amostras provavelmente aderideriram-se ao corpo das abelhas durante a coleta de néctar.

Em geral as abelhas têm maior especificidade em relação à coleta de pólen do que de néctar, podendo ser poliléticas (generalistas) ou oligoléticas, quando restringem suas coletas de pólen a uma ou poucas espécies de plantas relacionadas (Linsley 1958). Os resultados deste trabalho demonstram que as abelhas visitantes de Byrsonima chrysophylla não misturaram os recursos polínicos durante o vôo para a coleta de pólen, apresentando fidelidade às flores desta espécie de Malpighiaceae, o que, segundo Alves dos Santos (1998), é uma das características de polinizadores efetivos.

Apesar de o período seco parecer ser preferencial para a floração de comunidades de Malpighiaceae (Costa et al. 2006), a floração de Byrsonima chrysophylla ocorre no período chuvoso na região estudada, evidenciando a contribuição desta espécie vegetal na manutenção de recursos e sobrevivência dos seus visitantes florais.

A grande produção de flores durante várias semanas, observada em $B$. chrysophylla pode ser considerada uma estratégia que garante a atração de grande número de polinizadores (Gentry 1974), o que aumentaria a probabilidade de sucesso reprodutivo. Por outro lado, B. chrysophylla é uma das poucas fontes de óleo encontradas no local estudado (Silva et al. 2006) e uma importante fonte de recurso polínico utilizada por seus polinizadores na restinga de Barreirinhas, Maranhão.

\section{Agradecimentos}

A Profa. Léa Carreira, do Museu Paraense Emílio Goeldi, pelo auxílio na identificação dos tipos polínicos; a Marina Ramos, pela ajuda nas atividades de laboratório; a Fundação de Amparo à Pesquisa e ao Desenvolvimento Científico e Tecnológico do Maranhão - FAPEMA, pela bolsa de mestrado concedida à primeira autora; aos consultores anônimos, pelas valiosas sugestões.

\section{Referências}

Albuquerque, P.M.C. \& Rêgo, M.M.C. 1989. Fenologia das abelhas visitantes de murici (Byrsonima crassifolia, Malpighiaceae). Boletim do Museu Paraense Emílio Goeldi, Série Zoológica 5: 163-178.

Alves dos Santos, I. 1998. A importância das abelhas na polinização e manutenção da diversidade dos recursos vegetais. Pp. 101-106. In: Anais do III Encontro sobre Abelhas. Ribeirão Preto, USP.

Anderson, W.R. 1979. Floral conservatism in neotropical Malpighiaceae. Biotropica 11: 219-223.

Anderson, W.R. 1981. Malpighiaceae. In: The botany of the Guayana highland. Part XI. Memoirs of the New York Botanical Garden 32: 21-306.

Barros, M.A.G. 1992. Fenologia da floração, estratégias reprodutivas e polinização de espécies simpátricas do gênero Byrsonima Rich (Malpighiaceae). Revista Brasileira de Biologia 52: 343-353.

Benezar, R.M.C. \& Pessoni, L.A. 2006. Biologia floral e sistema reprodutivo de Byrsonima coccolobifolia (Kunth) em uma savana amazônica. Acta Amazônica 36: 159-168.

BRASIL. 1984. Instituto Brasileiro de Meio Ambiente e dos Recursos Naturais Renováveis (IBAMA). Secretaria do Estado do Meio Ambiente e Turismo do Maranhão (SEMATUR). Diagnóstico dos principais problemas ambientais do Estado do Maranhão. São Luís, Lithograf.

Buchmann, S.L. 1983. Buzz pollination in Angiosperms. Pp. 73-113. In: Handbook of experimental pollination biology (C.E. Jones \& R.J. Little, eds.). New York, Scientific and Academic Editions.

Buchmann, S.L. 1987. The ecology of oil flowers and their bees. Annual Review of Ecology and Systematics 18: 343-369.

Cameron, K.M.; Chase, M.W.; Anderson, W.R. \& Hills, H.G. 2001. Molecular systematics of Malpighiaceae: evidence from plastid $r b c L$ and $m a t K$ sequences. American Journal of Botany 88: 1847-1862. 
Costa, C.B.N.; Costa, J.A.S. \& Ramalho, M. 2006. Biologia reprodutiva de espécies simpátricas de Malpighiaceae em dunas costeiras da Bahia, Brasil. Revista Brasileira de Botânica 29: 103-114.

Eckert, J.E. 1942. The pollen required by a colony of honeybees. Journal of Economic Entomology 35: 309-311.

Erdtman, G. 1966. Pollen morphology and plant taxonomic. Stockholm, Almqvist \& Wilsell.

Freitas, B.M.; Alves, J.E.; Brandão, G.F. \& Araújo, Z.B. 1999 Pollination requirements of West Indian Cherry (Malpighia emarginata) and its putative pollinators, Centris bees, in NE Brazil. Journal of Agricultural Science 133: 303-311.

Gentry, A.H. 1974. Flowering phenology and diversity in tropical Bignoniaceae. Biotropica 6: 64-68.

Gerling, D.; Velthuis, W.H.D. \& Hefetz, A. 1989. Bionomics of the large carpenter bee of the genus Xylocopa. Annual Review of Entomology 34: 163-190.

IBGE (Instituto Brasileiro de Geografia e Estatística). 1984. Atlas do Maranhão. Rio de Janeiro, IBGE.

Linsley, E.G. 1958. The ecology of solitary bees. Hilgardia 27: 543-597.

Louveaux, J.; Maurizio, A. \& Vorwohl, G. 1978. Methods of melissopalynology. Bee World 59: 139-157.

Machado, I.C. 2004. Oil-collecting bees and related plants: a review of the studies in the last twenty years and case histories of plants occurring in NE Brazil. Pp. 255-279. In: B.M. Freitas (ed.). Solitary bees: conservation, rearing and management for pollination. Fortaleza, Imprensa Universitária.

MMA/IBAMA. 2002. Plano de manejo do Parque Nacional dos Lençóis Maranhenses - MA

Pedro, S.M.R. 1994. Interações entre abelhas e flores em uma área de cerrado no NE do Estado de São Paulo: abelhas coletoras de óleo (Hymenoptera: Apoidea: Apidae). Pp. 243-255. In: Anais do I Encontro sobre Abelhas. Ribeirão Preto, USP.

Ramalho, M. \& Silva, M. 2002. Flora oleífera e sua guilda de abelhas em uma comunidade de restinga tropical. Sitientibus série Ciências Biológicas 2: 34-43.

Rêgo, M.M.C. \& Albuquerque, P.M.C. 2004. O murici e suas abelhas. Ciência Hoje 208: 58-60.

Rêgo, M.M.C. \& Albuquerque, P.M.C. 2006. Polinização do murici. São Luís, Edufma.

Rêgo, M.M.C.; Albuquerque, P.M.C.; Ramos, M.C. \& Carreira, L.M. 2006. Aspectos da biologia de nidificação de Centris flavifrons (Friese) (Hymenoptera: Apidae, Centridini), um dos principais polinizadores do murici (Byrsonima crassifolia L. Kunth, Malpighiaceae), no Maranhão. Neotropical Entomology 35: 579-587.
Ribeiro, E.; Rêgo, M. \& Machado, I. 2006. Aspectos da polinização do murici pitanga - Byrsonima chrysophylla (Malpighiaceae). Pp. 49-54. In: M. Rêgo \& P. Albuquerque, eds. Polinização do Murici. São Luís, EDUFMA.

Schlindwein, C.; Wittmann, D.; Martins, C.F.; Hamm, A.; Siqueira, J.A.; Schiffler, D. \& Machado, I.C. 2005. Pollination of Campanula rapunculus L. (Campanulaceae): How much pollen flows into pollination and into reproduction of oligolectic pollinators? Plant Systematics and Evolution 250: 147-156.

Silva, O.; Rêgo, M. \& Albuquerque, P. 2006. Recursos florais disponíveis na restinga para os polinizadores do murici. Pp. 79-88. In: M. Rêgo \& P. Albuquerque, eds. Polinização do Murici. São Luís, EDUFMA.

Silveira F.A.; Melo, G.A.R. \& Almeida, E.A.B. 2002. Abelhas Brasileiras: Sistemática e Identificação. Belo Horizonte, Fundação Araucária.

Simpson, B.B. \& Neff, J.L. 1981. Floral rewards: alternatives to pollen and nectar. Annals of the Missouri Botanical Garden 68: 301-322.

Souza, V.C. \& Lorenzi, M.H. 2005. Botânica sistemática. Nova Odessa, Instituto Plantarum.

Teixeira, L.A.G. \& Machado, I.C. 2000. Sistema de polinização e reprodução de Byrsonima sericea DC (Malpighiaceae). Acta Botanica Brasilica 14: 347-357.

Vinson, S.B.; Williams, H.J.; Frankie, G.W. \& Shrum, G. 1997. Floral lipid chemistry of Byrsonima crassifolia (Malpighiaceae) and a use of floral lipids by Centris bees (Hymenoptera: Apidae). Biotropica 29: 76-83.

Vogel, S. 1974. Ölblumen und ölsammelnde Bienen. Akademie der Wissenchaften und der Literatur. Tropische und subtropische Pflanzenwelt 7. Franz. Steiner Verlag, Wiesbaden.

Vogel, S. 1990. History of the Malpighiaceae in the light of pollination ecology. Memoirs of The New York Botanical Garden 55: 130-142.

Wittmann, D. \& Schlindwein, C. 1995. Melittophilus Plants, their pollen and flower visiting bees in Southern Brazil. 1. Loasaceae. Biociências 3: 19-34.

Zanella, F.C.V. 2002. Sistemática, filogenia e distribuição geográfica das espécies sul-americanas de Centris (Paracentris) Cameron, 1903 e de Centris (Penthemisia) Moure, 1950, incluindo uma análise filogenética do 'grupo Centris' sensu Ayala, 1998 (Hymenoptera, Apoidea, Centridini). Revista Brasileira de Entomologia 46: 435-488. 